\title{
Empowering Voices, Building Bridges, Creating Solutions
}

\author{
D J Williams and Emily E. Prior \\ Editors
}

We are delighted to present the very first issue of the Journal of Positive Sexuality! We would like to thank our outstanding editorial board and our many volunteers at the Center for Positive Sexuality for their efforts in helping to make this new project a success. Our vision is for JPS to be an open, multidisciplinary forum where people from diverse social roles and positions can access and share quality, peer-reviewed information pertaining to any aspect of positive sexuality. We welcome submissions from academics, practitioners, administrators, students, and professionals from diverse backgrounds and disciplines on any topic pertaining to positive sexuality.

There has been considerable interest and curiosity about JPS, especially because it is not structured like a traditional academic journal. Articles are relatively short, cover a full range of topics and genres, and are written for a broad and diverse readership. Indeed, the purposes of JPS are to empower many voices, build bridges across professional contexts, and encourage people to work together to help create solutions to social problems involving sexuality. We hope that our concise, understandable articles will increase JPS readership and participation, while also promoting widespread practical application of sex-positive knowledge.

While sexuality is ubiquitous in Western society, much of our culture, particularly in the United States, reflects degrees of sex-negativity. Strong sex-negative attitudes often tend to empower some voices but silence many others, restrict communication about sexuality and sexual behavior, and marginalize those who do not conform to normalized sexual scripts. Popular sexnegative attitudes and beliefs among the masses play out daily in various social settings, including contributing to how our youth are educated about sexuality, how laws and policies are formed that govern sexual behavior, and how our society addresses and responds to sexual violence. These effects, realized or not, have substantial consequences to the lives of many citizens.

Many scholars have observed significant problems that have occurred from policies, however well-intentioned, that are rooted in sex-negativity. Abstinence-only sex-education among youth has been both costly and ineffective. Even comprehensive sex education often reflects salient cultural biases, while having little to say about healthy sexual identities and diverse sexual expression. At the same time, adults who enjoy alternative consensual sexual relationships and/or alternative sex practices continue to be at risk for marginalization and discrimination. A growing number of recent studies also show that current sex offender policies, rooted in widespread popular myths and moral panic, are often ineffective and sometimes seem to increase, rather than decrease, risk for recidivism. Laws have become sufficiently strict that the occasional youth who is "caught" sexting may end up in the criminal justice system and chronically labelled as a sex offender. 
The above examples illustrate major problems with laws and policies rooted in widespread sexnegativity, which is characterized by fear and the lack of open, honest communication. An important underlying questions is: How can a society expect to have socially-just, effective policies concerning sexuality when sexuality is not openly and honestly discussed in the first place? It cannot.

We recognize that sex-negativity and positivity are not dichotomous but seem to be opposite ends of a continuum. Furthermore, specific locations within a broader culture also seem to vary in this respect. Nevertheless, positive sexuality that is rooted in open and honest communication, human diversity, inclusion, compassion, self-determination and empowerment seems to hold promise in creating effective solutions to several contemporary social problems. Indeed, there is much to explore both theoretically and practically within positive sexuality, and there is tremendous potential for realizing many societal benefits that adventurous exploration may bring!

With this introduction, welcome to JPS! We hope you enjoy our current and future articles and that you find them useful within your personal and professional roles. We now invite you to join with us in empowering voices, building bridges, and creating solutions. 